\title{
A GAUSSIAN MEASURE FOR CERTAIN CONTINUED FRACTIONS
}

\author{
SOFIA KALPAZIDOU
}

\begin{abstract}
We solve a variant of Gauss' problem for grotesque continued fraction using the approach of dependence with complete connections.
\end{abstract}

1. Introduction. For a very readable account of Gauss' introduction of a measure to study ordinary continued fraction expansions, see [3]. In [7] Rieger introduced the grotesque continued fraction expansion for an irrational number $y$ of the interval $Y=[G-2, G]$, with $G=(\sqrt{5}+1) / 2$, as follows. Let $\varepsilon_{1}(y)=\operatorname{sign} y$. Then $\varepsilon_{1} y^{-1}$ can be written uniquely as $\varepsilon_{1} y^{-1}=\alpha_{1}(y)+T(y)$ with $\alpha_{1} \geqslant 1, \alpha_{1} \equiv 1(\bmod 2)$. Because $T(y) \in Y$ the above process can be inductively continued and as a consequence we get

$$
\begin{aligned}
y & =\frac{\varepsilon_{1}(y)}{\alpha_{1}(y)+T(y)}=\frac{\varepsilon_{1}(y)}{\alpha_{1}(y)+\frac{\varepsilon_{2}(y)}{\alpha_{2}(y)+\cdot}} \\
& =\left[\begin{array}{l}
\varepsilon_{1}(y), \varepsilon_{2}(y), \ldots \\
\alpha_{1}(y), \alpha_{2}(y), \ldots
\end{array}\right],
\end{aligned}
$$

where $\alpha_{n} \in N, \alpha_{n} \equiv 1(\bmod 2), \varepsilon_{n} \in\{-1,1\}, \alpha_{n}+\varepsilon_{n}>1, n=1,2, \ldots$ The expression (1.1) is called the grotesque continued fraction expansion of $y$. The iterated maps of $T$ are

$$
T^{n} y=\left[\begin{array}{c}
\varepsilon_{n+1}(y), \ldots \\
\alpha_{n+1}(y), \ldots
\end{array}\right], \quad n=1,2, \ldots
$$

with $T^{0} y=y$.

Let $\mu$ be a nonatomic probability measure on the $\sigma$-algebra of the Borel sets of $Y$ and define

$$
V_{n}(w)=V_{n}(w, \mu)=\mu\left(T^{n}<w\right), \quad n=1,2, \ldots,
$$

with $V_{0}(w)=\mu([G-2, w))$. Here, using the approach of dependence with complete

Received by the editors November 6, 1984.

1980 Mathematics Subject Classification. Primary 28Dxx; Secondary 60A10, 60Gxx.

(C)1986 American Mathematical Society $0002-9939 / 86 \$ 1.00+\$ .25$ per page 
connections, we find the limit

$$
\lim _{n \rightarrow \infty} \mu\left(T^{n}<w\right)=1
$$

and estimate the error $\left(T^{n}<w\right)-1$ to be $O\left(q^{n}\right)$. Our result generalizes and improves that which Rieger obtained using another method (see [8]) for $\mu=(1 / 2) \lambda$, where $\lambda$ is the Lebesgue measure, with convergence rate of $O\left(q^{\sqrt{n}}\right)$. Throughout we need the notation

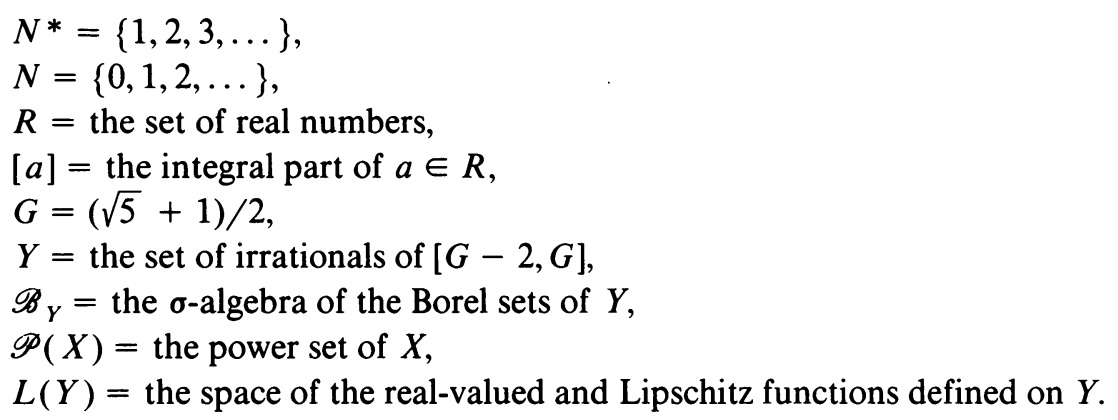

2. The Gauss-type relation. In the following we require the definitions and terminology of $[2,4$ and 5$]$. Define

$$
X=\left\{(x, \varepsilon): x \in N^{*}, x \equiv 1(\bmod 2),|\varepsilon|=1, x+\varepsilon>1\right\} .
$$

Then we have

Proposition 2.1 (GAUSS-TYPE RELATION). For each $n \in N, V_{n}$ satisfies the relation

$$
V_{n+1}(w)=\sum_{(x, \varepsilon) \in X} \varepsilon\left(V_{n}\left(\frac{\varepsilon}{x+G-2}\right)-V_{n}\left(\frac{\varepsilon}{x+w}\right)\right), \quad w \in[G-2, G] .
$$

Proof. We remark that

$$
T^{n}=\frac{\varepsilon_{n+1}}{\alpha_{n+1}+T^{n+1}}
$$

Therefore

$$
\begin{aligned}
V_{n+1}(w)= & \mu\left(\frac{1}{\alpha_{n+1}+w}<T^{n}<\frac{1}{\alpha_{n+1}+G-2}\right) \\
& +\mu\left(\frac{-1}{\alpha_{n+1}+G-2}<T^{n}<\frac{-1}{\alpha_{n+1}+w}\right)
\end{aligned}
$$

and the desired result follows.

We now assume that $V_{0}^{\prime}$ exists and is bounded ( $\mu$ has bounded density). By induction we have that $V_{n}^{\prime}, n \in N^{*}$, exist and are bounded as well. By differentiating the above Gauss-type equation we obtain

$$
V_{n+1}^{\prime}(w)=\sum_{(x, \varepsilon) \in X} \frac{1}{(x+w)^{2}} V_{n}^{\prime}\left(\frac{\varepsilon}{x+w}\right)
$$


Let us introduce the functions $f_{n}$ in (2.1) where

$$
f_{n}(w)=\frac{V_{n}^{\prime}(w)}{\rho(w)}, \quad n \in N,
$$

with $\rho(w)=1 /(w+1), w \in[G-2, G]$. Then (2.1) becomes

$$
f_{n+1}(w)=\sum_{(x, \varepsilon) \in X} \frac{w+1}{(w+x)(w+x+\varepsilon)} f_{n}\left(\frac{\varepsilon}{x+w}\right) .
$$

Proposition 2.2. The function

$$
P(w,(x, \varepsilon))=\frac{w+1}{(w+x)(w+x+\varepsilon)}
$$

defines a transition probability function from $\left(Y, \mathscr{B}_{Y}\right)$ to $(X, \mathscr{P}(X))$.

Proof. We have to verify that

$$
\sum_{(x, \varepsilon)} P(w,(x, \varepsilon))=1
$$

for all $w \in Y$. To this end we write the left side of (2.2) as

$$
(w+1) \sum_{x=1,3,5, \ldots}\left[\left(\frac{1}{w+x}-\frac{1}{w+x+1}\right)+\sum_{x=3,5, \ldots}\left(\frac{1}{w+x-1}-\frac{1}{w+x}\right)\right]
$$

and the desired equality follows.

Proposition 2.2 allows us to consider the random system with complete connections (RSCC) (see [2])

$$
\{(Y, \mathscr{Y}),(X, \mathscr{X}), u, P\}
$$

where

$$
\begin{gathered}
\mathscr{Y}=\mathscr{B}_{Y}, \quad \mathscr{X}=\mathscr{P}(X), \quad u(w,(x, \varepsilon))=\frac{\varepsilon}{x+w}, \\
P(w,(x, \varepsilon))=\frac{w+1}{(w+x)(w+x+\varepsilon)} .
\end{gathered}
$$

Further, we denote by $U$ the associated Markov operator of the RSCC (2.3) with the transition probability function $Q(\cdot, \cdot)$. Then $Q^{n}(\cdot, \cdot)$ will be the transition probability function after $n$ paths of the same Markov chain (see [4]).

3. The ergodicity of the random system. Let us consider the norm $\|\cdot\|_{L}$ defined on $L(Y)$ by

$$
\|f\|_{L}=\sup _{y \in Y}|f(y)|+\sup _{y^{\prime} \neq y^{\prime \prime}} \frac{\left|f\left(y^{\prime}\right)-f\left(y^{\prime \prime}\right)\right|}{\left|y^{\prime}-y^{\prime \prime}\right|}, \quad f \in L(Y) .
$$

As is well known, $\left(L(Y),\|\cdot\|_{L}\right)$ is a Banach algebra. The following proposition can be proved in the same manner as in [5].

PROPOSITION 3.1. The RSCC (2.3) is uniformly ergodic. 
Moreover, Theorem 2.1.57 of Iosifescu-Theodorescu [2] implies that $Q^{n}(\cdot, \cdot)$ converges uniformly to a probability $Q^{\infty}$ and that there exist two positive constants $q<1$ and $c$ such that

$$
\left\|U^{n} f-U^{\infty} f\right\|_{L} \leqslant c q^{n}
$$

for all $n \in N^{*}, f \in L(Y)$, where

$$
U^{n} f(\cdot)=\int_{Y} f(y) Q^{n}(\cdot, d y), \quad U^{\infty} f=\int_{Y} f(y) Q^{\infty}(d y) .
$$

By virtue of Lemma 2.1.58 of Iosifescu-Theodorescu [2], $U$ has no eigenvalues of modulus 1 other than 1 .

Let $B(Y, \mathscr{Y})$ be the Banach space of all real-valued bounded and $\mathscr{Y}$-measurable functions defined on $Y$ with the uniform norm $|\psi|=\sup _{y \in Y}|\psi(y)|$. Then, taking into account Proposition 2.1.6 of [2], the adjoint $U^{*}$ of the operator $U$ (with the transition probability function $Q(\cdot, \cdot))$ in $B(Y, \mathscr{Y})$ is

$$
\left(U^{*} \mu\right)(\cdot)=\int_{Y} Q(y, \cdot) \mu(d y),
$$

where $\mu$ belongs to the Banach space of all bounded additive set functions on $\mathscr{Y}$ with bounded variation with the norm given by the total variation.

By virtue of Proposition 3.1, Theorem 3.3.3 of [2] says that $Q^{\infty}$ is the only eigenvector of $U^{*}$ and as a consequence

$$
\int_{G-2}^{G} Q(y, B) Q^{\infty}(d y)=Q^{\infty}(B)
$$

for all the Borel sets of $Y$.

Further, we determine the form of $Q^{\infty}$.

Proposition 3.2. The probability $Q^{\infty}$ has the density

$$
\rho(w)=\frac{1}{w+1}, \quad w \in[G-2, G]
$$

with the normalizing factor $1 /(3 \log G)$.

Proof. We have

$$
Q(y, B)=\sum_{\substack{(x, \varepsilon) \in X \\ \varepsilon(x+y)^{-1} \in B}} P(y,(x, \varepsilon)),
$$

for all $y \in Y$ and $B \in \mathscr{B}_{Y}$. By virtue of uniqueness of $Q^{\infty}$ we have to show that the measure with density $\rho$ defined in (3.4) satisfies the equality (3.3). Because the intervals $[u, G], 0 \leqslant u<G$, and $[G-2, v], G-2<v \leqslant 0$, generate $\mathscr{B}_{Y}$, it suffices to verify the equality (3.3) only for $B=[u, G]$ and $B=[G-2, v]$. 
Let us consider $B=[u, G], 0 \leqslant u<G$. Suppose first that $\left[u^{-1}\right]$ is an even number. Then

$$
\begin{array}{rl}
\int_{G-2}^{G} & Q(y,[u, G]) \rho(y) d y=\int_{G-2}^{G}\left(\sum_{\substack{x \equiv 1(\bmod 2) \\
u \leqslant(x+y)^{-1} \leqslant G}} P(y,(x, 1))\right) \rho(y) d y \\
= & \int_{G-2}^{G}\left(\sum_{\substack{x \equiv 1(\bmod 2) \\
1 \leqslant x \leqslant\left[u^{-1}-G\right]}} P(y,(x, 1))\right) \rho(y) d y \\
= & \int_{u^{-1}-\left[u^{-1}\right]-1}^{G}\left(\sum_{\substack{x=1 \\
x \equiv 1(\bmod 2)}}^{\left[u^{-1}\right]-1} P(y,(x, 1))\right) \rho(y) d y \\
& +\int_{G-2}^{u^{-1}-\left[u^{-1}\right]-1}\left(\sum_{\substack{x=1 \\
x \equiv 1(\bmod 2)}}^{\left[u^{-1}\right]+1}(y,(x, 1))\right) \rho(y) d y \\
= & \int_{u^{-1}-\left[u^{-1}\right]-1}^{G}\left(\frac{1}{y+1}-\frac{1}{y+2}+\cdots+\frac{1}{y+\left[u^{-1}\right]-1}-\frac{1}{y-\left[u^{-1}\right]}\right) d y \\
& +\int_{G-2}^{u^{-1}-\left[u^{-1}\right]-1}\left(\frac{1}{y+1}-\frac{1}{y+2}+\cdots+\frac{1}{y+\left[u^{-1}\right]+1}-\frac{1}{y+\left[u^{-1}\right]+2}\right) d y \\
= & \log \frac{G u^{-1}}{(G-1)\left(u^{-1}+1\right)}=\log \frac{G+1}{u+1}=\int_{u}^{G} \rho(y) d y .
\end{array}
$$

If $\left[u^{-1}\right]$ is odd we obtain

$$
\begin{aligned}
\int_{G-2}^{G} Q(y,[u, G]) \rho(y) d y=\int_{G-2}^{u^{-1}-\left[u^{-1}\right]}\left(\sum_{\substack{x=1 \\
x=1(\bmod 2)}}^{\left[u^{-1}\right]} P(y,(x, 1))\right) \rho(y) d y \\
\quad+\int_{u^{-1}-\left[u^{-1}\right]}^{G}\left(\sum_{x=1}^{\left[u^{-1}\right]-2} P(y,(x, 1))\right) \rho(y) d y \\
=\int_{G-2}^{u^{-1}-\left[u^{-1}\right]}\left(\frac{1}{y+1}-\frac{1}{y+2}+\cdots+\frac{1}{y+\left[u^{-1}\right]}-\frac{1}{y+\left[u^{-1}\right]+1}\right) d y \\
\quad+\int_{u^{-1}-\left[u^{-1}\right]}^{G}\left(\frac{1}{y+1}-\frac{1}{y+2}+\cdots+\frac{1}{y+\left[u^{-1}\right]-2}-\frac{1}{y+\left[u^{-1}\right]-1}\right) d y \\
=\log \frac{G+1}{u+1}=\int_{u}^{G} \rho(y) d y .
\end{aligned}
$$

Finally, the proof for $B=[G-2, v], G-2<v \leqslant 0$, is similar.

4. The Gauss-type theorem. Now we are prepared to find the limit of $\mu\left(T^{n}<w\right)$ as $n \rightarrow \infty$ and the rate of convergence. 
Proposition 4.1. If $\mu$ has a Riemann integrable density, then

$$
\lim _{n \rightarrow \infty} \mu\left(T^{n}<w\right)=\frac{1}{3 \log G} \log (G(w+1)), \quad w \in[G-2, G] .
$$

If the density of $\mu$ is a Lipschitz function, then there exist two positive constants $c$ and $q<1$ such that for all $w \in[G-2, G], n \in N^{*}$,

$$
\mu\left(T^{n}<w\right)=\frac{1}{3 \log G}\left(1+\theta q^{n}\right) \log (G(w+1)),
$$

where $\theta=\theta(\mu, n, w)$ with $|\theta| \leqslant c$.

Proof. If $V_{0}^{\prime}$ is a Lipschitz function, then $f_{0} \in L(Y)$. By virtue of (3.2) we have

$$
U^{\infty} f_{0}=\int_{G-2}^{G} f_{0}(y) Q^{\infty}(d y)=\frac{1}{3 \log G} .
$$

Taking into account (3.1) there exist two constants $c$ and $q<1$ such that

$$
\left\|U^{n} f_{0}-U^{\infty} f_{0}\right\|_{L} \leqslant c q^{n} \text { for all } n \in N^{*} .
$$

Further, consider $C(Y)$ to be the metric space of real continuous functions defined on $Y$ with the norm $|\cdot|=\sup |\cdot|$. As $L(Y)$ is a dense subset of $C(Y)$ we have

$$
\lim _{n \rightarrow \infty}\left|\left(U^{n}-U^{\infty}\right) f_{0}\right|=0
$$

for all $f_{0} \in C(Y)$. Therefore (4.1) remains valid for measurable $f_{0}$ that are $Q^{\infty}$. almost surely continuous, that is, for Riemann-integrable $f_{0}$.

We have

$$
\begin{aligned}
\lim _{n \rightarrow \infty} \mu\left(T^{n}<w\right) & =\lim _{n \rightarrow \infty} \int_{G-2}^{w} U^{n} f_{0}(u) \cdot \rho(u) d u=\frac{1}{3 \log G} \int_{G-2}^{w} \rho(u) d u \\
& =\frac{1}{3 \log G} \log \frac{w+1}{G-1}=\frac{1}{3 \log G} \log (G(w+1)) .
\end{aligned}
$$

Corollary 4.2. Let $m \in N^{*},\left(i_{1}, j_{1}\right), \ldots,\left(i_{m}, j_{m}\right) \in X$ be fixed and take

$$
\mu(\cdot)=\frac{1}{2} \lambda\left(\cdot \mid \alpha_{r}=i_{r}, \varepsilon_{r}=j_{r}, r=1, \ldots, m\right) .
$$

Then

$$
\lambda\left(T^{n+m}<w \mid a_{r}=i_{r}, \varepsilon_{r}=i_{r}, r=1, \ldots, m\right)=\left(1+\theta_{m} q^{n}\right) \frac{2}{3 \log G} \log (G(w+1))
$$

where $\theta_{m}=\theta_{m}\left(n, w, s_{m}\right)$ with $s_{r}=j_{r}\left(i_{r}+s_{r-1}\right)^{-1}, r=1, \ldots, m, s_{0}=0$.

RemarK. The study of optimality of the rate of convergence in Proposition 4.1 remains an open question. Concerning this, Wirsing's method for the classical Gauss' problem cannot be applied in our case because the corresponding operator is not a positive one, see [10].

\section{REFERENCES}

1. S. Grigorescu and M. Iosifescu, Dependence with complete connections and applications, Ed. Stiintifica \& Encicloped., Bucharest, 1982.

2. M. Iosifescu and R. Theodorescu, Random processes and learning, Springer-Verlag, New York, 1969. 
3. M. Kac, Statistical independence in probability analysis, and number theory, Carus Math. Monograph, vol. 12, Math. Assoc. of Amer., Washington, D.C., 1959.

4. S. Kalpazidou, Some asymptotic results on digits of the nearest integer fraction, J. Number Theory (to appear).

5. On a random system with complete connections associated with the continued fraction to the nearer integer expansion, Rev. Roumaine Math. Pures Appl. 7 (1985), 527-537.

6. M. F. Norman, Markov processes and learning models, Academic Press, New York, 1972.

7. G. J. Rieger, Ein Hilbronn-Satz für Kettenbrüche mit ungeraden Teilnernnern, Math. Nachr. 101 (1981), 295-307.

8. On the metrical theory of continued fractions with odd partial quotients, in print.

9. F. Schweiger, Continued fractions with odd and even partial quotients, Arbeitsbericht of Math. Instit. der Univ. Salzburg 4 (1982), 59-70.

10. E. Wirsing, On the theorem of Gauss-Kusmin-Lévy and a Frobenius-type theorem for function spaces, Acta Arith. 24 (1974), 507-528.

Department of Mathematics, Faculty of Sciences, Aristotle University of Thessaloniki, THESSALONIKI, GREECE 